\title{
Nested models of relative judgment: Applications to a similarity averaging model
}

\author{
ROBERT F. FAGOT \\ University of Oregon, Eugene, Oregon 97403
}

\begin{abstract}
A theoretical analysis of the Eisler and Ekman (1959) model of similarity judgments for unidimensional continua is presented, based on a general model of relative judgment. This general model assumes that judgments are mediated by perceived relations of pairs of stimuli, that there exists a transformation of the judgmental response that is a function of the sensory ratio of the two stimuli, and that response bias operates in a multiplicative manner. Three structural conditions are presented, each imposing constraints on the structure of observed judgments. The structural conditions define three nested models of relative judgment, with the second a weakened version of the first, and the third a weakened version of the second. The special virtue of the general model is that it is applicable to a variety of judgmental tasks (e.g., ratio estimation, similarity, pair comparison), the key being derivation of the response transformation conforming to the structural conditions. The structural conditions thus constitute necessary conditions for several different judgmental models. The theory was first applied with success to ratio estimation judgments (Fagot, 1978), and this paper applies the general model to the Eisler and Ekman similarity "averaging" model. Empirical tests were carried out on published data for pitch, darkness, visual area, and heaviness judgments. Although the strong form of the model presented by Eisler and Ekman was rejected, weakened versions were generally supported by the data. These results were similar to those obtained for ratio estimation (Fagot, 1978), and are interpreted to be very promising for the general model of relative judgment.
\end{abstract}

Eisler and Ekman (1959) have proposed an appealingly simple model of similarity judgments for unidimensional continua, namely,

$$
\mathrm{S}_{\mathrm{ab}}=\frac{\psi_{\mathrm{a}}}{\left(\psi_{\mathrm{a}}+\psi_{\mathrm{b}}\right) / 2}=\frac{2 \psi_{\mathrm{a}} / \psi_{\mathrm{b}}}{\left(\psi_{\mathrm{a}} / \psi_{\mathrm{b}}\right)+1}, \quad\left(\psi_{\mathrm{a}} \leqslant \psi_{\mathrm{b}}\right)
$$

where $\mathrm{S}_{\mathrm{ab}}$ is the judged similarity between the two percepts and $\psi$ is a scaling function. Similarity is defined such that $0 \leqslant S_{a b} \leqslant 1$, with $S_{a b}=1$ for "perfect" similarity or identity, and $S_{a b}=0$ for perfect dissimilarity. Equation 1 thus assumes that the judged similarity of two stimuli is the ratio of the subjective magnitude of the smaller stimulus to the arithmetic mean of the magnitudes of the two stimuli.

It should be noted that although the $S_{a b}$ are interpreted as similarity judgments, Equation 1 is not a general similarity model in the sense used by most investigators who try to account for similarity judgments of stimuli varying on several dimensions. Equation 1 is applicable only to those situations in which stimuli vary with respect to a single attribute, such as pitch, brightness, heaviness, etc.

Various studies have made different interpretations of $\psi$. To distinguish these, we refer to Equation 1

Work on this paper was carried out, in part, during the author's tenure as a Fellow at the Netherlands Institute for Advanced Study, Wassenaar, Holland. as a similarity scaling model only when $\psi$ is interpreted as a similarity scale such that scale values may be inferred from the observed similarity judgments $S_{a b}$. Equation 1, as a scaling model, will be referred to as a similarity averaging model (SAM). On the other hand, if $\psi$ represents a scale derived from some other scaling method, e.g., ratio estimation, Equation 1 will be referred to as a similarity function.

A choice between these two interpretations cannot be made easily on the basis of previous empirical studies Ekman, Engen, Kunnapas, and Lindman (1964) and Ekman and Sjöberg (1965) accepted the relation given by Equation 1 as established, based on empirical studies by Eisler (1960), Eisler and Ekman (1959), and Ekman, Goude, and Waern (1961). However, the latter two studies treated Equation 1 strictly as a similarity function, and although Eisler and Ekman derived a similarity scale from Equation 1, and reported a good fit of the similarity scale to a scale derived from fractionation, there was no attempt to test Equation 1 as a scaling model. Tests of Equation 1 treated as a similarity function may have no direct bearing on the adequacy of this model equation as a scaling model. This will be clearer after the theoretical analysis presented in the next section, but the point follows from the fact that the two interpretations entail possibly different scaling functions. In the interpretation of Equation 1 as a scaling 
model, the scale $\psi$ is derived from similarity judgments $S_{a b}$, whereas in its interpretation as a similarity function, the scale $\psi$ is derived from some other scaling method, for example, ratio estimation. Thus, if Equation 1 is rejected (or accepted) with $\psi$ a ratio estimation scale, this result bears on the issue of the relationship betwen similarity judgments and ratio estimation judgments, but has no necessary bearing on whether a similarity scale $\psi$ exists, satisfying Equation 1 (the scaling model interpretation). Necessary conditions for the existence of a similarity scale $\psi$ satisfying Equation 1 are presented in the next section, and are expressed entirely in terms of observed similarity judgments $S_{a b}$.

Sjobberg (1966) carried out the first test of Equation 1 interpreted as a scaling model, based on data from judgments of pitch, darkness, visual area, and heaviness, presented in Eisler (1960), Eisler and Ekman (1959), and Ekman, Goude, and Waern (1961). Sjoberg concluded that the data did not satisfy Equation 1. The theoretical basis of Sjoberg's tests must await some theory to be presented later. However, it should be pointed out at this stage that, in spite of Sjoberg's conclusions, the data cited above will be interpreted in the present paper as generally supportive of Equation 1 as a fundamental similarity scaling model provided response biases are admitted (Fagot, 1978).

As suggested above, the difficulty in evaluating Equation 1 based on prior research has been the failure in most cases to distinguish between a similarity function and a similarity scaling model, and the lack of a theoretical analysis of Equation 1 as a scaling model. The key question to pose in interpreting this equation as a scaling model is: What relations among observed similarity judgments are entailed by Equation 1 if $\psi$ is a similarity scaling function? The answer would provide a set of necessary conditions on observed $S_{a b}$ if a similarity ratio scale $\psi$ exists satisfying Equation 1.

Fagot (1978) posed a similar question for alternative ratio estimation models: i.e., what relations must hold among observed ratio estimations if a given model is assumed. He presented three structural conditions, each placing constraints on the structure of ratio estimation data. The main theoretical contribution of the present paper is to show how the Fagot (1978) theory of relative judgment can be extended to the SAM. The theory will then be tested against the published data cited above.

\section{THEORY}

The ratio estimation theory (Fagot, 1978) applies to reported ratio judgments of stimulus a to stimulus $b$ (relative to a defined attribute) denoted by $R_{a b}$, and assumes that the response $R_{a b}$ is a function of sensory ratios $\psi_{\mathrm{a}} / \psi_{\mathrm{b}}$ and possibly bias parameters, i.e., $\mathbf{R}_{\mathrm{ab}}=\mathbf{f}\left(\psi_{\mathrm{a}} / \psi_{\mathrm{b}}, \beta_{\mathrm{b}}\right)$. Three specific model equations were considered:

$$
\begin{aligned}
& \mathbf{R}_{\mathrm{ab}}=\psi_{\mathrm{a}} / \psi_{\mathrm{b}}, \\
& \mathbf{R}_{\mathrm{ab}}=\beta \psi_{\mathrm{a}} / \psi_{\mathrm{b}}, \\
& \mathbf{R}_{\mathrm{ab}}=\beta_{\mathrm{b}} \psi_{\mathrm{a}} / \psi_{\mathrm{b}},
\end{aligned}
$$

where $\beta$ and $\beta_{b}$ may be interpreted as bias parameters and $\psi$ is the scaling function. ${ }^{1}$ Equations 2,3 , and 4 are referred to as the classical (C), constant bias (CB), and relative bias (RB) models, respectively. The bias parameter could account for variations due to experimental arrangements, such as position or time-order effect.

Three structural conditions, each imposing constraints on the structure of the observed ratio estimates, were presented:

$$
\begin{array}{ll}
\text { Ratio consistency (C1): } & \mathbf{R}_{\mathrm{ac}}=\mathbf{R}_{\mathrm{ab}} \mathbf{R}_{\mathrm{bc}} \\
\text { Product constancy (C2): } & \mathbf{R}_{\mathrm{ab}} \mathbf{R}_{\mathrm{bd}}=\mathbf{R}_{\mathrm{ac}} \mathbf{R}_{\mathrm{cd}} \\
\text { Ratio constancy (C3): } & \mathbf{R}_{\mathrm{ad}} / \mathbf{R}_{\mathrm{bd}}=\mathbf{R}_{\mathrm{ac}} / \mathbf{R}_{\mathrm{bc}}
\end{array}
$$

It can be shown that Equation 2-the $\mathrm{C}$ modelentails $\mathrm{C} 1, \mathrm{C} 2$, and $\mathrm{C} 3$ (but $\mathrm{C} 2$ and $\mathrm{C} 3$ are entailed by $\mathrm{C} 1$ ); Equation $3-$ the $\mathrm{CB}$ model-entails $\mathrm{C} 2$ and C3; and Equation 4-the RB model-entails C3. A further condition, monotonicity (see Fagot, 1978), is entailed by each of the three models.

The bias parameters in Equations 3 and 4 are expressed entirely in terms of observables:

$$
\beta=\mathbf{R}_{\mathrm{ab}} \mathbf{R}_{\mathrm{bc}} / \mathbf{R}_{\mathrm{ac}}
$$

and

$$
\beta_{\mathrm{b}}=\mathbf{R}_{\mathrm{ab}} \mathbf{R}_{\mathrm{bc}} / \mathbf{R}_{\mathrm{ac}} \quad(\mathrm{b}=2,3, \ldots, \mathrm{n}-1) .
$$

Not all three structural conditions need to be satisfied to justify scale construction. Given that monotonicity is satisfied: if $\mathrm{Cl}$ is satisfied, then Equation 2 can be used to construct a ratio scale. If $\mathrm{C} 1$ is violated but $\mathrm{C} 2$ and $\mathrm{C} 3$ are satisfied, then Equation 3 can be used to construct a ratio scale with $\beta$ estimated from Equation 8. Finally, if $\mathrm{C} 1$ and $\mathrm{C} 2$ are violated but $\mathrm{C3}$ is satisfied, then Equation 4 can be used to construct a ratio scale with $\beta_{\mathrm{b}}$ estimated from Equation 9.

Thus, the structural conditions define nested models of relative judgment. Starting with the weakest model (RB) (i.e., weakest in the sense that it places weakest constraints on the structure of the data), successive strengthening is obtained by adding on 
conditions one at a time, such that a weaker model is nested within a stronger model (RB is nested within $\mathrm{CB}$, and $\mathrm{CB}$ within $\mathrm{C}$ ).

A common procedure for testing scaling models has been to estimate scale values, usually by a least squares procedure, and test goodness-of-fit of observed data (e.g., responses $R_{a b}$ ) to values reproduced using the estimated scale values. The disadvantage of this approach is the very large number of parameters used (scale values), and the obscuring of systematic trends in the data. By contrast, the structural conditions are parameter-free conditions that are necessary for the existence of the scaling function $\psi$ satisfying the model equation (Equation 2, 3 , or 4). There is the added benefit that violation of a structural condition uncovers systematic errors.

The value of the $C B$ and $R B$ models might be questioned on the grounds that the addition of parameters simply results in more mathematical freedom to account for data. However, comparative tests of the models (including the $\mathrm{C}$ model) would be carried out on the structural conditions, which, as noted above, are parameter-free. Hence, the bias parameters in the CB and RB models do not gain mathematical freedom to account for data in the usual sense, since these parameters are not used in goodness-of-fit tests. Fagot (1978) showed that C1 was generally violated for ratio estimation data, a finding justifying abandonment of the classical theory of ratio estimation. However, the $\mathrm{CB}$ and RB models salvage the classical theory that the response is a function of the sensory ratio by the simple revision of further assuming that response bias operates in a multiplicative fashion producing two further weakened versions of the classical model.

The theory of relative judgment summarized above will now be applied to the SAM (Equation 1) through introduction of bias parameters.

The SAM given by Equation 1 may be written in the form

$$
\psi_{\mathrm{a}} / \psi_{\mathrm{b}}=\mathrm{S}_{\mathrm{ab}} /\left(2-\mathrm{S}_{\mathrm{ab}}\right),
$$

and defining $S_{a b}^{\prime}=S_{a b} /\left(2-S_{a b}\right)$, we have

$$
\mathrm{S}_{\mathrm{ab}}^{\prime}=\psi_{\mathrm{a}} / \psi_{\mathrm{b}}
$$

Equation 11 is of the same mathematical form as Equation 2; and hence, since Equation 2 entails that $\mathrm{C} 1, \mathrm{C} 2$, and $\mathrm{C} 3$ hold for $\mathrm{R}_{\mathrm{ab}}$, Equation 11 entails that $\mathrm{C} 1, \mathrm{C} 2$, and $\mathrm{C} 3$ hold for similarity transforms $S_{a b}^{\prime}$ (i.e., substitute $S_{a b}^{\prime}$ for $R_{a b}$ in Equations 5, 6, and 7). Equation 11 will be referred to as the classical similarity averaging model (SAM-C).

The theory of relative judgment assumes that stimulus a is judged relative to stimulus $b$, that the response is a function of the sensory ratio $\psi_{\mathrm{a}} / \psi_{\mathrm{b}}$, and that bias operates in a multiplicative manner (Equations 2, 3, and 4). Hence, applying these assumptions to judgments of similarity and the SAM given by Equatiion 11, we get

$$
\mathrm{S}_{\mathrm{ab}}^{\prime}=\alpha \psi_{\mathrm{a}} / \psi_{\mathrm{b}}
$$

and

$$
\mathrm{S}_{\mathrm{ab}}^{\prime}=\alpha_{\mathrm{b}} \psi_{\mathrm{a}} / \psi_{\mathrm{b}},
$$

where $\alpha$ and $\alpha_{b}$ permit different magnitude biases for ratio estimation and similarity judgments. Equations 12 and 13 are equivalent to Equation 1 complicated by the multiplicative operation of bias on $\psi_{\text {a }}$; i.e., the similarity judgment of stimulus a relative to stimulus b converts $\psi_{\mathrm{a}}$ in Equation 1 to $\alpha \psi_{\mathrm{a}}$ entailing Equation 12, and to $\alpha_{\mathrm{b}} \psi_{\mathrm{a}}$ entailing Equation 13.

Equation 12 is of the same mathematical form as Equation 3, and hence Equation 12 entails that $\mathrm{C2}$ and C3 (but not C1) hold for similarity transforms $\mathrm{S}_{\mathrm{ab}}^{\prime}$. Equation 12 defines the constant bias similarity averaging model (SAM-CB).

Equation 13 is of the same mathematical form as Equation 4, and hence Equation 13 entails that $\mathrm{C3}$ (but not $\mathrm{C} 1$ and $\mathrm{C2}$ ) holds for similarity transforms $\mathrm{S}_{\mathrm{ab}}^{\prime}$. Equation 13 defines the relative bias similarity averaging model (SAM-RB).

Thus it is seen that $\mathrm{C1}, \mathrm{C} 2$, and $\mathrm{C} 3$ provide directly testable consequences of the SAMs expressed entirely in terms of observed similarity transforms $\mathrm{S}_{\mathrm{ab}}^{\prime}$.

The similarity bias parameters $\alpha$ and $\alpha_{\mathrm{b}}$ are expressed entirely in terms of observed similarity transforms $S_{a b}^{\prime}$ as given by Equations 8 and 9, respectively, with $S_{a b}^{\prime}$ substituted for $R_{a b}$.

The theory of relative judgment described above can be stated in more general terms, as follows, for some judgment $\mathrm{J}_{\mathrm{ab}}$ :

$$
\mathrm{f}\left(\mathrm{J}_{\mathrm{ab}}\right)=\mathrm{g}\left(\Theta_{\mathrm{b}}\right) \psi_{\mathrm{a}} / \psi_{\mathrm{b}},
$$

where $\theta_{b}$ is the bias parameter. The function $g$ determines the model: $\mathrm{g}\left(\Theta_{\mathrm{b}}\right)=1$ entails the $\mathrm{C}$ model, $g\left(\theta_{b}\right)=\theta$ entails the CB model, and $g\left(\theta_{b}\right)=$ $\Theta_{b}$ entails the RB model. The function $f$ determines the response transformation conforming to the structural conditions. To apply the theory to a given scaling model, it is only necessary to derive the response transformation $\mathrm{f}$ satisfying Equation 14 . For example, for the SAM, $f\left(S_{a b}\right)=S_{a b} / 2-S_{a b}$ $=\mathrm{S}_{\mathrm{a} b}^{\prime}$, leading to Equations 11,12 , and 13 as special cases of Equation 14 applicable to the SAM.

As another example, consider the probability model

$$
\mathrm{P}_{\mathrm{ab}}=\mathrm{V}_{\mathrm{a}} /\left(\mathrm{V}_{\mathrm{a}}+\mathrm{V}_{\mathrm{b}}\right)
$$


(Bradley \& Terry, 1952; Luce, 1959), where $P_{a b}$ is the probability that stimulus $a$ is chosen to stimulus $b$ and $\mathrm{V}$ is a scaling function. The response transformation is $P_{a b}^{\prime}=P_{a b} / 1-P_{a b}$, and $g\left(\theta_{b}\right)=1$, i.e., $P_{a b}^{\prime}=V_{a} / V_{b}$. Hence, a necessary condition for the existence of a scaling function $V$ satisfying Equation 15 is that the response transformation $\mathrm{P}_{\mathrm{ab}}^{\prime}$ conforms to $\mathrm{Cl}$, which is a well-known testable consequence of Equation 15. Furthermore, $f\left(\theta_{b}\right)=\theta, \theta_{b}$ provides extensions of this theory that require satisfaction of $\mathrm{C} 2, \mathrm{C} 3$ for $\mathrm{P}_{\mathrm{ab}}^{\prime}$, and sets of data may exist for which $\mathrm{C} 1$ is violated and $\mathrm{C} 3$ or $\mathrm{C} 2$ and $\mathrm{C} 3$ are satisfied.

\section{Related Scaling Theory}

Sjöberg (1966) points out that scaling theories generally assume strict perceptual invariance, i.e., that only one perceptual magnitude corresponds to each stimulus. This assumption is embodied in Equations 2, 3, and 4 for ratio estimation and Equations 1, 11,12 , and 13 for similarity judgments. Sjöberg (1966) proposes as an alternative a variable/standard (VS) model that assigns a different scale value to a stimulus depending on its status as a comparison (variable) or standard stimulus. The Sjöberg model for ratio estimation is simply

$$
\mathrm{R}_{\mathrm{ab}}=\mathrm{V}_{\mathrm{a}} / \mathrm{S}_{\mathrm{b}}
$$

and his version of the SAM is

$$
\mathrm{S}_{\mathrm{ab}}^{\prime}=\mathrm{V}_{\mathrm{a}}^{\prime} / \mathrm{S}_{\mathrm{b}}^{\prime},
$$

where $V_{a}$ and $V_{a}^{\prime}$ refer to the scale values of stimulus $a$ as a variable stimulus, and $S_{b}$ and $S_{b}^{\prime}$ refer to the scale values for stimulus $b$ as a standard stimulus.

It can be shown that the Sjöberg VS model and the RB model are data equivalent, i.e., they are consistent with exactly the same finite sets of basic "observation"' statements (Adams, Fagot, \& Robinson, 1970). Specifically, the VS model entails C3 (but not $\mathrm{Cl}$ or $\mathrm{C} 2$ ). Equation 16 entails that

$$
\mathrm{V}_{\mathrm{b}} / \mathrm{S}_{\mathrm{b}}=\mathbf{R}_{\mathrm{ab}} \mathbf{R}_{\mathrm{bc}} / \mathbf{R}_{\mathrm{ac}},
$$

and hence $V_{b} / S_{b}=\beta_{b}$, the bias parameter for the ratio estimation RB model; and Equation 17 entails that

$$
\mathrm{V}_{\mathrm{b}}^{\prime} / \mathrm{S}_{\mathrm{b}}^{\prime}=\mathrm{S}_{\mathrm{ab}}^{\prime} \mathrm{S}_{\mathrm{bc}}^{\prime} / \mathrm{S}_{\mathrm{ac}}^{\prime},
$$

and hence $V_{b}^{\prime} / S_{b}^{\prime}=\alpha_{b}$, the bias parameter for the SAM-RB.

Furthermore, if the $\mathrm{C}$ model is correct, then $\mathrm{V}_{\mathrm{b}}^{\prime} / \mathrm{S}_{\mathrm{b}}^{\prime}=1$ for all $\mathrm{b}$; and if the CB model is correct, then $V_{b}^{\prime} / S_{b}^{\prime}=\alpha$ for all $b$.

Classical psychophysical scaling models (e.g., ratio estimation and magnitude estimation) consider a response to be a direct reaction to the stimulus, i.e., that subjects' judgments are mediated by sensations evoked by single stimuli. The view expressed in the relative judgment theory is that subjects judge pairs of stimuli, not single stimuli, a view consistent with relation theory as formulated by Krantz (1972), based on a proposal by R.N. Shepard that subjects' judgments are mediated by perceived relations of pairs of stimuli. Similarity is an obvious property of pairs of stimuli, and for ratio estimation, the perceived relation is interpreted to be sensation ratios. Unlike relation theory, the theory of relative judgment formulated in the present paper accounts for biased judgments due to the interactive effect of stimuli. A flaw in Krantz' elegant theory is his incorporation of $\mathrm{Cl}$ as an axiom. For ratio estimation, $\mathrm{Cl}$ has been shown to fail for a variety of perceptual attributes (Fagot, 1978).

\section{Judgment-Judgment (J-J) Relations}

Cliff (1973) distinguishes three basically different types of scales: magnitude scales, category scales, and discrimination scales. Magnitude scales are based on "ratio methods," such as magnitude estimation, fractionation, ratio estimation, and magnitude production. Much research has been carried out on scale relations, including a very extensive study by Stevens and Galanter (1957) on the relation between ratio and category scales. Of interest in the present paper is the class of methods generating "magnitude scales" and in particular the relation between ratio estimation magnitude scales and similarity scales based on the SAMs, including the corresponding $J-J$ relations between $R_{a b}$ and $S_{a b}$ or $S_{a b}^{\prime}$. The SAM scales have in common with magnitude scales a reliance on direct numerical estimation and the production of ratio scales provided consistency tests are satisfied. Do SAM scales belong to the class of magnitude scales? If the answer is yes, then a SAM scale should be related to a ratio estimation scale by a similarity transformation, i.e., should be identical except for possibly different units. Denoting the similarity (SAM) scale by $\psi_{\mathrm{s}}$ and the ratio estimation scale by $\Psi_{\mathrm{r}}$, the scale relation hypothesis is that $\psi_{\mathrm{s}}=\mathrm{K} \psi_{\mathrm{r}}$, where $\mathrm{K}$ is a positive constant corresponding to change of unit.

The scale relation hypothesis will be expressed in terms of a judgment-judgment (J-J) relation, i.e., a functional relationship between ratio estimates $R_{a b}$ and similarity transforms $S_{\mathrm{ab}}^{\prime}$. A J-J relation corresponds to a psychosensory relation as defined by Marks (1974).

$\mathrm{J}-\mathrm{J}$ relations are derived assuming that one of the bias models (C, CB, or RB) holds for both $R_{a b}$ and $\mathrm{S}_{\mathrm{ab}}^{\prime}$. Assuming $\psi_{\mathrm{s}}=\mathrm{K} \psi_{\mathrm{r}}$, the following results hold: 
(1) If the $C$ model holds for both $R_{a b}$ and $S_{a b}^{\prime}$, then

$$
\mathrm{S}_{\mathrm{ab}}^{\prime}=\mathbf{R}_{\mathrm{ab}}
$$

(2) if the CB model holds for both $R_{a b}$ and $S_{a b}^{\prime}$, then

$$
\mathrm{S}_{\mathrm{ab}}^{\prime}=(\alpha / \beta) \mathrm{R}_{\mathrm{ab}}
$$

(3) if the RB model holds for both $R_{a b}$ and $S_{a b}^{\prime}$, then

$$
S_{a b}^{\prime}=\left(\alpha_{b} / \beta_{b}\right) R_{a b} .
$$

Note that even if both types of judgment are biased, the simple $\mathrm{J}-\mathrm{J}$ relation given by Equation 20 holds if the bias magnitudes are equal $(\alpha=\beta$ in Equation 21 or $\alpha_{\mathrm{b}}=\beta_{\mathrm{b}}$ for all $\mathrm{b}$ in Equation 22).

Equations 20,21 , and 22 place strong constraints on the relation between similarity and ratio estimates. Note that in testing the fit of Equations 21 and 22 there are no free parameters, since $\alpha, \beta$ in Equation 21 and $\alpha_{\mathrm{b}}, \beta_{\mathrm{b}}$ in Equation 22 are independently estimated from their respective judgment experiments and operate as known constants.

Kunnapas and Kunnapas $(1973,1974)$ assumed that similarity judgments were a power function of empirical ratio estimations:

$$
\mathrm{S}_{\mathrm{ab}}=\mathrm{kR}_{\mathrm{ab}}^{\mathrm{n}} \text {. }
$$

It is clear that Equation 23 postulates a $\mathrm{J}-\mathrm{J}$ relation, not a scaling model, and hence is not a logical comparator to the SAMs.

Equation 23 does, however, suggest that the similarity and ratio estimation scales may not be equivalent, except for change of unit, as assumed in deriving Equations 20,21, and 22. If we assume that the similarity scale $\psi_{\mathrm{s}}$ is a power function of the ratio estimation scale $\psi_{\mathrm{r}}$, i.e., $\psi_{\mathrm{s}}=\phi \psi_{\mathrm{r}}^{\gamma}$, then the RB model entails that

$$
\mathrm{S}_{\mathrm{ab}}^{\prime} / \alpha_{\mathrm{b}}=\left(\mathrm{R}_{\mathrm{ab}} / \beta_{\mathrm{b}}\right)^{\gamma},
$$

where $\gamma$ is the only parameter to be estimated. For the CB model, Equation 24 is modified by the sub- stitutions $\alpha_{\mathrm{b}}=\alpha, \beta_{\mathrm{b}}=\beta$; and for the C model, by $\alpha_{b}=\beta_{b}=1$.

\section{Tests of Structural Conditions for the SAMs}

Monotonicity and the three structural conditions were tested on similarity transforms $S_{a b}^{\prime}$ by using data from published studies for four attributespitch, darkness, visual area, and heaviness. Experimental procedures for these studies are described briefly in Kunnapas and Kunnapas (1973), and references to the original studies are given in Table 1.

Monotonicity was perfectly satisfied for each of the four attributes. Ratio estimation judgments were also made by subjects for three of the four attributesdarkness, visual area, and heaviness-with no violations of monotonicity observed (Fagot, 1978).

Each structural condition was tested in the form $\mathrm{Y}=\mathrm{X}$, where $\mathrm{Y}$ and $\mathrm{X}$ are the two sides of the equation for each condition (e.g., for $\mathrm{C} 1, \mathrm{Y}=$ $\mathrm{S}_{\mathrm{ac}}^{\prime}$ and $\mathrm{X}=\mathrm{S}_{\mathrm{ab}}^{\prime} \mathrm{S}_{\mathrm{bc}}^{\prime}$ ). Each condition was analyzed in two ways: (1) agreement, in the sense of reliability, between $\mathrm{Y}$ and $\mathrm{X}$ as measured by the intraclass correlation coefficient (ICC) (Bartko, 1976); and (2) tests for the presence of systematic errors in the plot of $Y$ as a function of $X$. Simple $t$ tests for paired differences, $Y_{i}-X_{i}$, were carried out to test for the presence of systematic errors. For further details on the analysis, see Fagot (1978). ${ }^{2}$

The ICCs and results of the tests for the presence of systematic errors are presented in Table 1.

Figures 1-4 give plots of each structural condition on similarity transforms for each of the four studies. In each plot, the dotted line is the identity line $\mathrm{Y}=\mathrm{X}$.

Table 1 shows that for all four attributes the reliability is appreciably lower for $\mathrm{Cl}$, and that reliabilities for $\mathrm{C} 2$ and $\mathrm{C} 3$ tend to be quite high.

In general, the tests for systematic errors in Table 1 and Figures 1-4 are encouraging for $\mathrm{C} 2$ and $\mathrm{C} 3$ but fail to support $\mathrm{Cl}$ and hence the SAM-C. In particular: (1) For darkness, the TSE was significant for all three structural conditions, but Figure 2 shows very marked systematic errors for $\mathrm{C} 1$, yet only slight systematic error for $\mathrm{C} 2$ and $\mathrm{C} 3$ with a reasonably close fit to the identity lines. (2) The TSE rejected $\mathrm{C} 1$ in three cases, but not for visual area. But note

\begin{tabular}{|c|c|c|c|c|c|c|c|}
\hline \multirow[b]{2}{*}{ Attribute } & \multirow[b]{2}{*}{ Reference } & \multicolumn{3}{|c|}{ ICC } & \multicolumn{3}{|c|}{ TSE } \\
\hline & & $\mathrm{Cl}$ & $\mathrm{C} 2$ & $\mathrm{C} 3$ & $\mathrm{Cl}$ & $\mathrm{C} 2$ & C3 \\
\hline Pitch & Eisler \& Ekman (1959) & .854 & .898 & .924 & $\dagger$ & $*$ & $* *$ \\
\hline Darkness & Ekman, Goude, \& Waern (1961) & .856 & .987 & .988 & $+\dagger$ & $\dagger$ & $* *$ \\
\hline Visual Area & Ekman, Goude, \& Waem (1961) & .888 & .979 & .946 & * & $*$ & $*$ \\
\hline Heaviness & Eisler (1960) & .932 & .986 & .988 & $\dagger \dagger$ & * & * \\
\hline
\end{tabular}
the marked systematic deviations for $\mathrm{Cl}$ in Figure 3, not detected by the TSE with only 5 degrees of

Table 1

Intraclass Correlation Coefficient (ICC) and Tests for Systematic Error (TSE)

*Nonsignificant. $\quad * * p<.05 . \quad \dagger_{p}<.01 . \quad \dagger+p<.001$. 


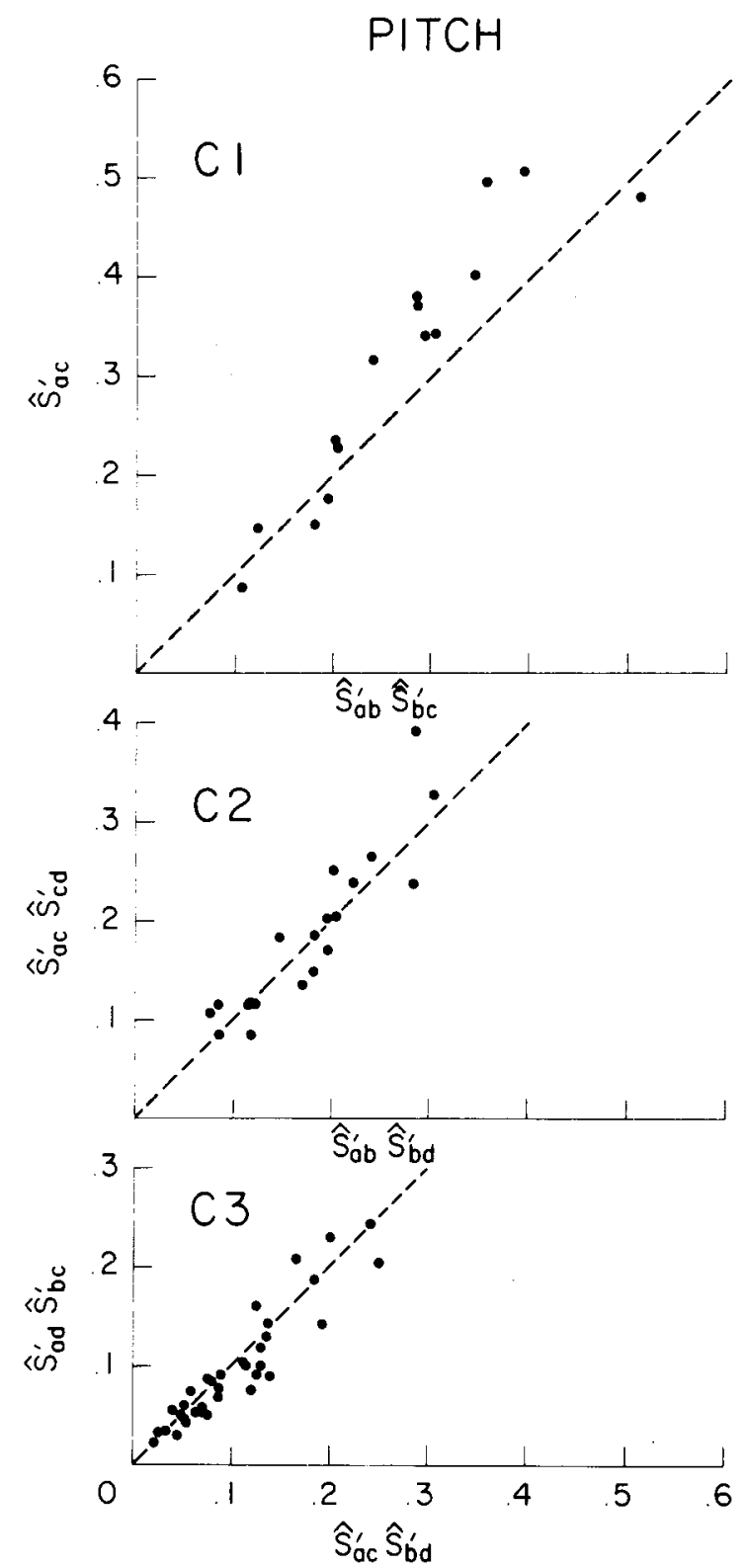

Figure 1. Comparative fit of structural conditions for pitch similarity transforms (Eisler \& Ekman, 1959).

freedom. Figure 3 shows a good fit for $\mathrm{C} 2$ and $\mathrm{C} 3$. (3) The TSE for heaviness was highly significant for $\mathrm{C} 1$ but not significant for $\mathrm{C} 2$ and $\mathrm{C} 3$. Figure 4 shows very marked systematic errors for $\mathrm{Cl}$ but good fits and absence of systematic errors for $\mathrm{C} 2$ and $\mathrm{C} 3$. (4) Figures 1-4 show the same pattern: large systematic errors for $\mathrm{Cl}$ with substantially improved fits and reductions of systematic errors for $\mathrm{C} 2$ and $\mathrm{C} 3$. Except for pitch, systematic errors for $\mathrm{Cl}$ are in the same direction $\left(\mathrm{S}_{\mathrm{ab}}^{\prime} \mathrm{S}_{\mathrm{b} c}^{\prime}>\mathrm{S}_{\mathrm{ac}}^{\prime}\right)$ and in the same direction observed for ratio estimation for judgments of nine attributes, including darkness, visual area, and heaviness (Fagot, 1978). The reversal in the case of pitch is perplexing.
In general, the tests of the structural conditions are interpreted as providing a firm basis for rejecting the SAM-C in the form proposed by Eisler and Ekman (1959), but as giving moderately good support for the bias forms of the SAM as given by Equations 12 and 13.
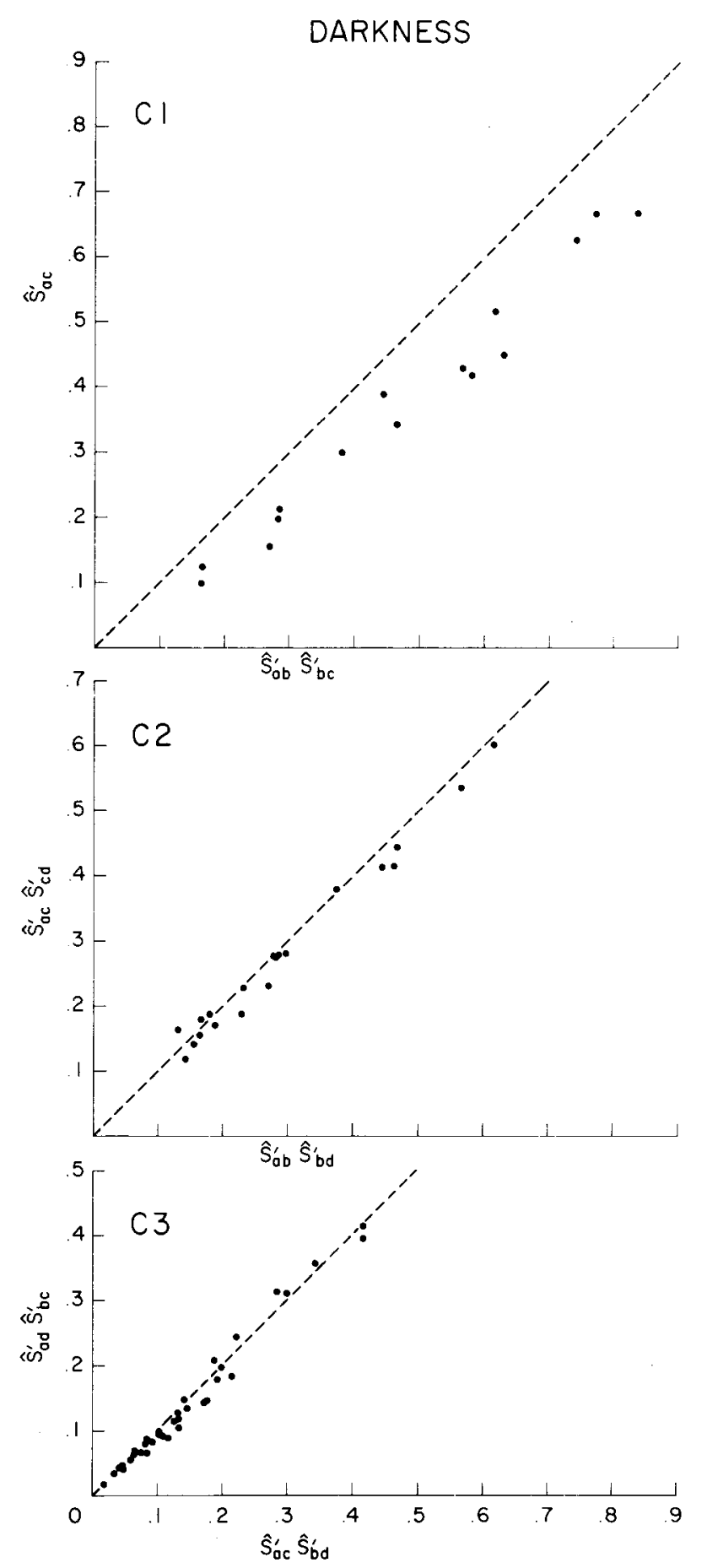

Figure 2. Comparative fit of structural conditions for darkness similarity transforms (Ekman, Goude, \& Waern, 1961 ). 


\section{VISUAL AREA}

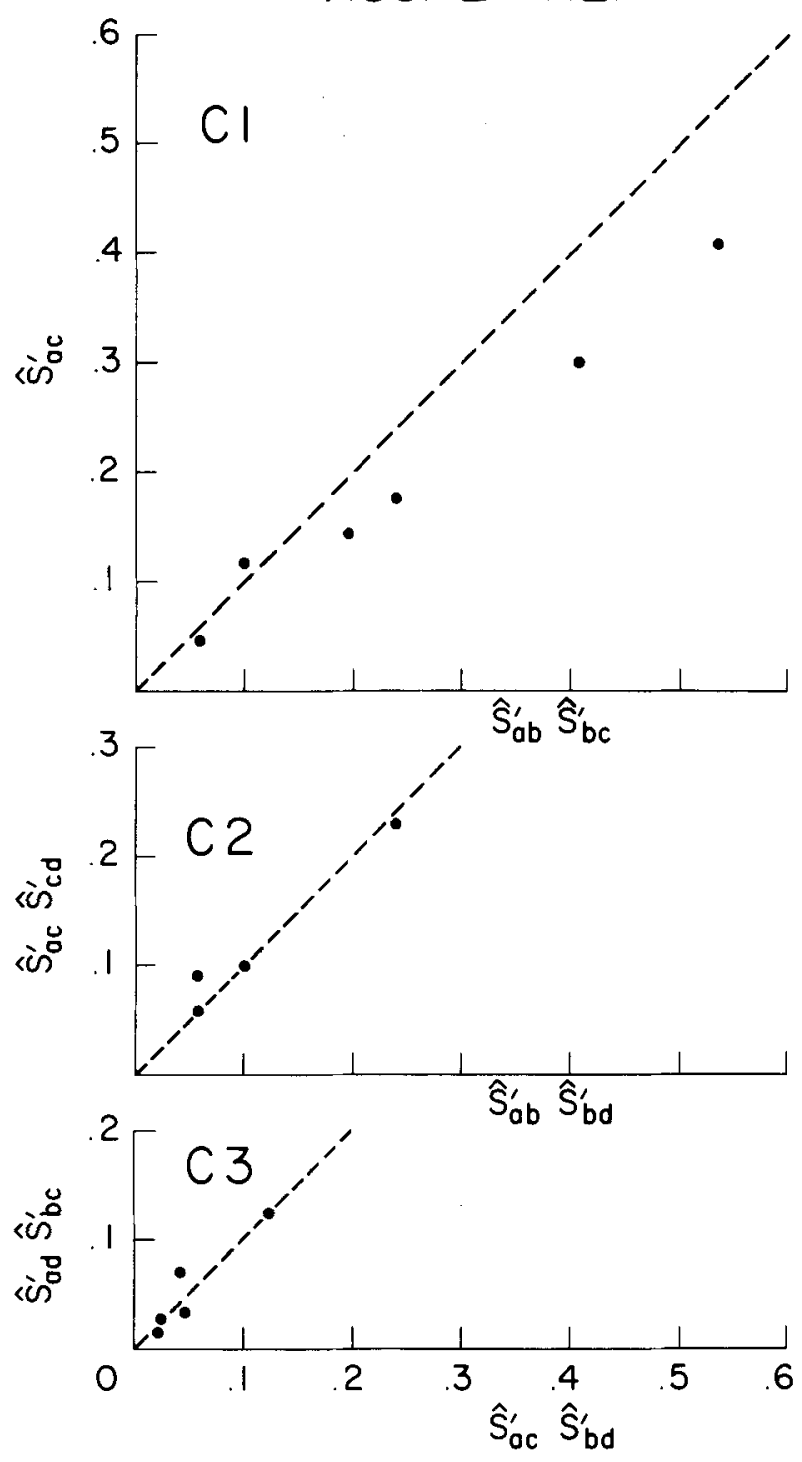

Figure 3. Comparative fit of structural conditions for visual area similarity transforms (Ekman, Goude, \& Waern, 1961).

\section{Tests of J-J Relations}

Tests of the J-J relations were carried out for darkness, visual area, and heaviness, but not for pitch, since for the latter the method of fractionation was used, providing estimates of theoretical $\psi_{\mathrm{a}} / \psi_{\mathrm{b}}$, not empirical ratio estimates $R_{a b}$.

Table 2 presents results of the regression analysis of $S_{a b}^{\prime}$ on $R_{a b}$. Note that for the regression model $Y=b_{1} X+b_{0}$ the hypothesis $H_{0}: b_{1}=1$ was rejected in all three cases; hence, the simple $\mathrm{J}-\mathrm{J}$ relation entailed by the $C$ model (Equation 20) must be rejected.

The hypothesis $\mathrm{H}_{0}: \mathrm{b}_{0}=0$ was not rejected for darkness and visual area; hence, for these two attributes, regression through the origin is tenable.
Turning to the results for the regression model $Y=b X$, we see that the estimate of the slope $(\hat{b})$ of the best-fitting line through the origin is estimated by $\alpha / \beta$ extremely well-the error is .002 and .003 for darkness and visual area, respectively.

Plots of $S_{a b}^{\prime}$ as a function of $R_{a b}$ for darkness and visual area are given in Figures 5 and 6, respectively. The dotted line is the function $S_{\mathrm{ab}}^{\prime}=$

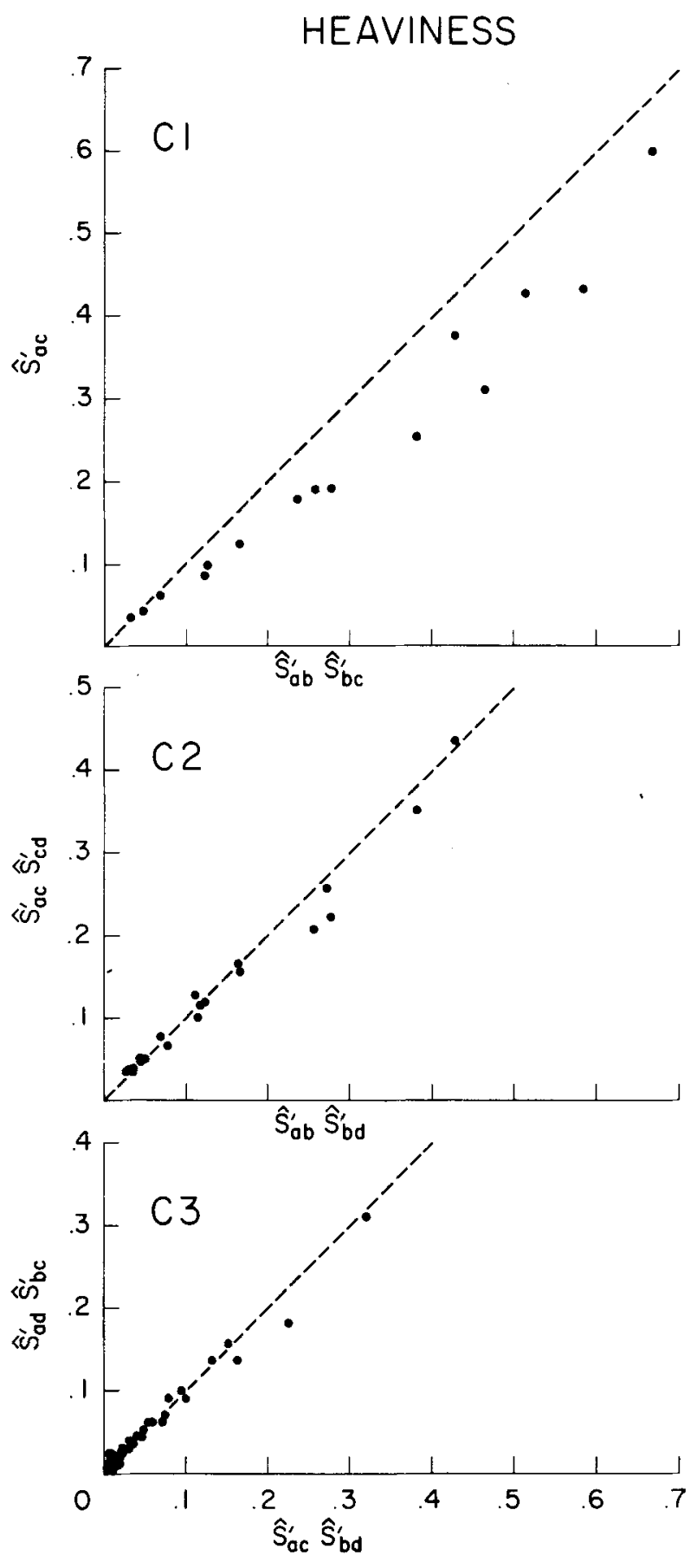

Figure 4. Comparative fit of structural conditions for heaviness similarity transforms (Eisler, 1960). 
Table 2

Regression of $S_{a b}^{\prime}(Y)$ on $R_{a b}(X)$

\begin{tabular}{|c|c|c|c|c|c|c|}
\hline \multirow[b]{4}{*}{ Attribute } & \multicolumn{6}{|c|}{ Regression Model } \\
\hline & \multicolumn{4}{|c|}{$Y=b_{1} X+b_{0}$} & & \\
\hline & \multirow[b]{2}{*}{$\hat{b}_{0}$} & \multirow[b]{2}{*}{$\hat{b}_{1}$} & \multicolumn{2}{|c|}{$\mathrm{H}_{\mathrm{o}}:$} & \multicolumn{2}{|c|}{$\mathrm{Y}=\mathrm{bX}$} \\
\hline & & & $b_{1}=1$ & $b_{0}=0$ & $\hat{b}$ & $\alpha / \beta$ \\
\hline Darkness & .010 & .954 & $+\dagger$ & * & .969 & .971 \\
\hline Visual Area & -.018 & .954 & $\dagger \dagger$ & * & .921 & .924 \\
\hline Heaviness & -.043 & .897 & $+\dagger$ & $t$ & .830 & .918 \\
\hline
\end{tabular}

*Nonsignificant. $\quad t p<.01 . \quad t+p<.001$.

$(\alpha / \beta) \mathrm{R}_{\mathrm{ab}}$ entailed by the CB model (Equation 21 ). Note that the fit is, in each case, very good without any indication of systematic error.

These results support the $\mathrm{J}-\mathrm{J}$ relation entailed by the $\mathrm{CB}$ model for darkness and visual area. The fits are particularly impressive considering the fact that the slope $(\alpha / \beta)$ was determined in independent scaling experiments and not estimated via the regression model.

For a test of the $\mathrm{J}-\mathrm{J}$ relation entailed by the $\mathrm{RB}$ model (Equation 22), plots (not presented) of $\mathrm{S}_{\mathrm{ab}}^{\prime} /$ $\alpha_{b}$ as a function of $R_{a b} / \beta_{b}$ were constructed. The fits for darkness and visual area were somewhat better than given in Figures 5 and 6 for the CB model, but further analysis of the RB model is not necessary since the stronger constraints placed on the $\mathrm{J}-\mathrm{J}$ relation by the $\mathrm{CB}$ model are supported.

Note that the results in Table 2 are not supportive for heaviness: as already indicated, $\mathrm{H}_{0}: \mathrm{b}_{1}=1$ is rejected; $H_{0}: b_{0}=0$ is also rejected, and $\alpha / \beta$ deviates appreciably from $\hat{b}$. The plot of $S_{a b}^{\prime}$ as a function of $R_{a b}$ is presented in Figure 7. Note that all the

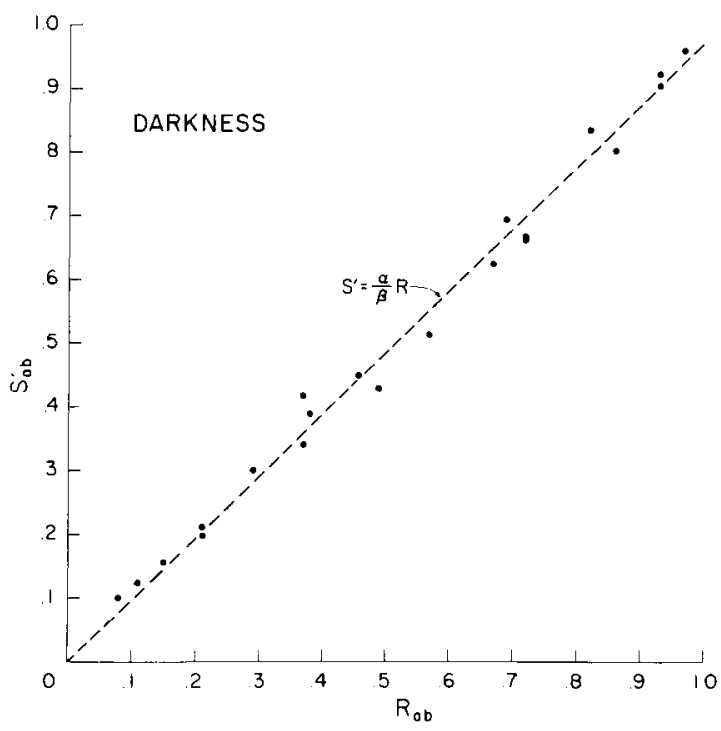

Figure 5. Graph of the J-J relation for darkness (Ekman, Goude, \& Waern, 1961) entailed by the CB model (Equation 21). points lie below the dotted line $\mathrm{S}^{\prime}=(\alpha / \beta) \mathrm{R}$, exhibiting large systematic errors. The results for heaviness may be due to the untenability of the assumption that the similarity and ratio estimation scales are equivalent except for change of unit. Therefore, the power function hypothesis embodied in Equation 24 was tested for the CB model in log form:

$$
\log \left(\mathrm{S}_{\mathrm{ab}}^{\prime} / \alpha\right)=\gamma \log \left(\mathrm{R}_{\mathrm{ab}} / \beta\right) .
$$

Figure 8 shows a plot of $\log \left(\mathrm{S}_{\mathrm{ab}}^{\prime} / \alpha\right)$ as a function of $\log \left(R_{a b} / \beta\right)$, where the dotted line is the identity line. The fit is very appreciably better than that of Figure 7, giving some support to the hypothesis that for heaviness, unlike the cases of darkness and visual area, the scales are related by a power function.

\section{DISCUSSION}

The authors of the studies listed in Table 1 accepted Equation 1 as established, based on their

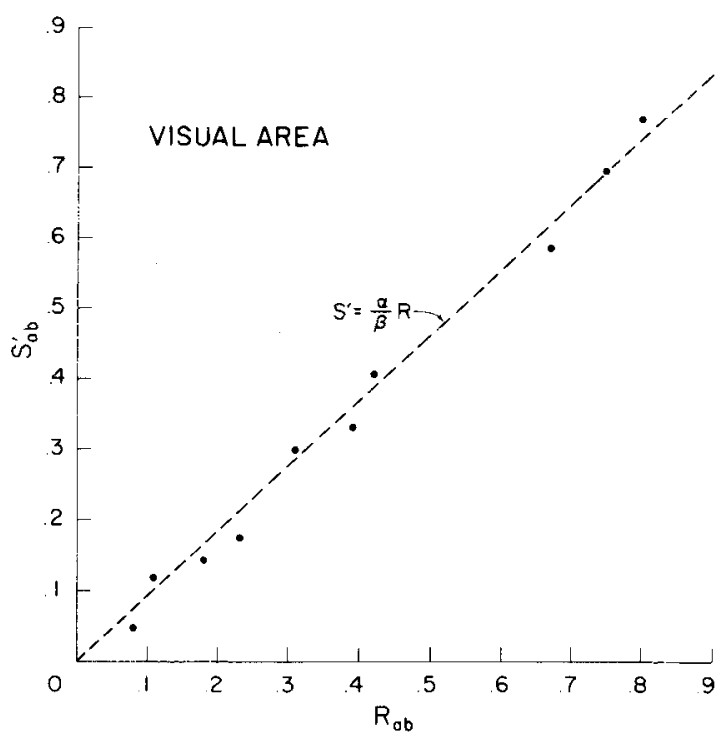

Figure 6. Graph of the J-J relation for visual area (Ekman, Goude, \& Waern, 1961) entailed by the CB model (Equation 21). 


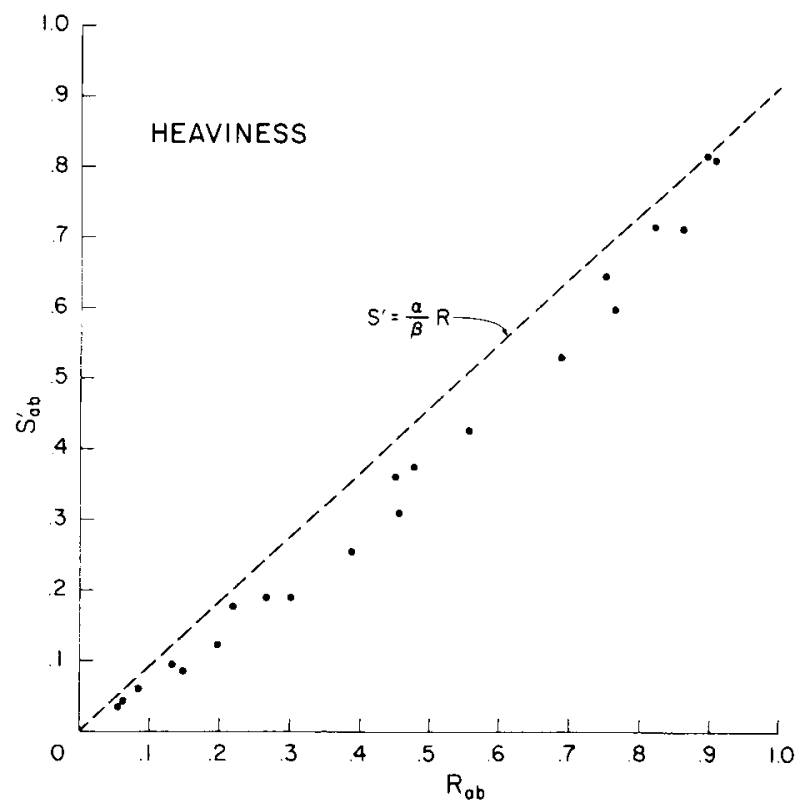

Figure 7. Graph of the J-J relation for heaviness (Fisler, 1960) entailed by the CB model (Equation 21).

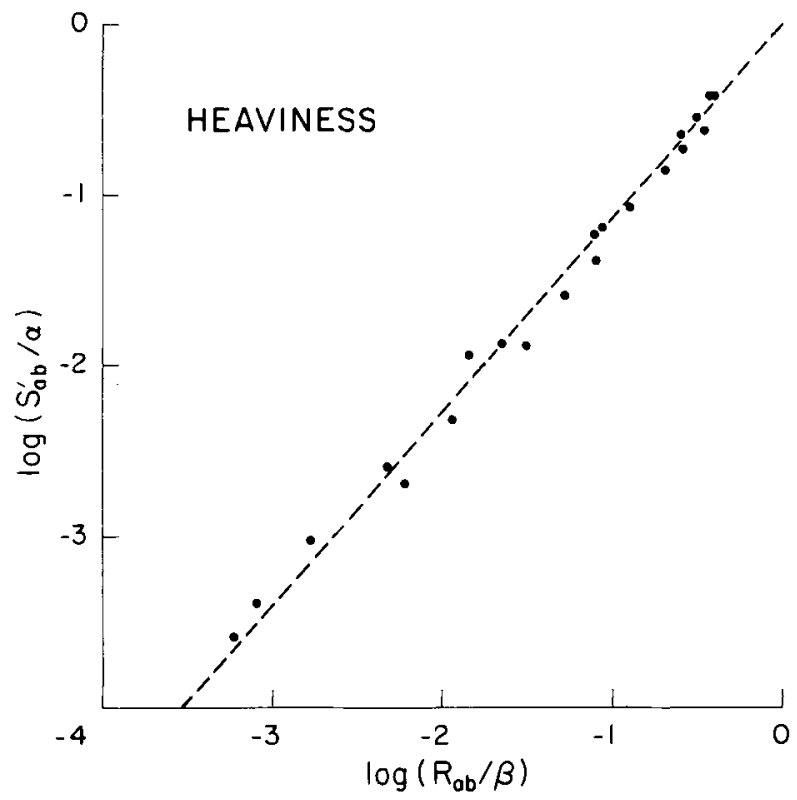

Figure 8. Graph of the $\log -\log$ form of the $\mathrm{J}-\mathrm{J}$ relation for heaviness (Eisler, 1960) entailed by the CB model under the assumption that the similarity and ratio estimation scales are related by a power function (Equation 25 ).

data for pitch, darkness, visual area, and heaviness. Yet Sjöberg (1966) rejected Equation 1 based on his analysis of these data; Sjöberg (1971) rejected Equation 1 for new data for judgments of circular area and heaviness; and Franzén, Nordmark, and Sjöberg (Note 1) also rejected Equation 1 based on pitch judgments. These inconsistent conclusions are based in part on a failure to distinguish between Equation 1 interpreted as a similarity function and as a scaling model. In the following discussion, we begin with the evaluation of the SAMs as scaling models.

Among the early studies cited, only Sjöberg (1966) carried out an empirical test of Equation 1 interpreted as a scaling model. Sjöberg based his tests on the VS model, and deduced that if Equation 1 was correct, then $\mathrm{V} / \mathrm{S}=1$; i.e., each stimulus has only one scale value (perceptual invariance). Using this test, Sjöberg rejected Equation 1 for all four sets of data listed in Table 1. However, as shown in the section on Theory, $\mathrm{V} / \mathrm{S}=1$ is a constraint only for SAM-C, and hence the Sjöberg analysis does not provide a basis for rejecting the similarity bias models (SAM$\mathrm{CB}$ and SAM-RB). Sjöberg's rejection of Equation 1 is paralleled in the present paper by the rejection of $\mathrm{C1}$, and both analyses agree that the SAM-C is an inadequate similarity model. But the reasonable conformity of the four sets of data to C2 and C3 illustrated in Figures 1-4 support the tenability of the similarity bias models.

Similar results were obtained for ratio estimation, particularly the clear rejection of C1 (Fagot, 1978). The results for ratio estimates and similarity judgments support the general judgment model given by Equation 14 , but indicate clearly that $g\left(\theta_{b}\right) \neq 1$.

Four other studies tested Equation 1 as a similarity function for ratio estimation and similarity judgments. These included Eisler (1960), for heaviness; Ekman, Goude, and Waern (1961), for darkness and visual area; Sjobberg (1971), for circular area and heaviness; and Franzén, Nordmark, \& Sjöberg (Note 1) for pitch. Data from the latter two studies are not available to the author, but the analyses for both studies led to the rejection of Equation 1 as a similarity function with $\psi$ interpreted as a ratio estimation scale. Furthermore, the darkness, visual area, and heaviness data (Table 1) are clearly inconsistent with the simple $\mathrm{J}-\mathrm{J}$ relation given by Equation 20. Of course, Equation 1 cannot be unequivocally rejected as a similarity function since it is possible that the relation may hold if $\psi$ is a scale derived from some method other than ratio estimation.

Going beyond the analyses made in the studies cited above, we see that Figures 5 and 6 show reasonably good support for the CB-model form of the J-J relation (Equation 21) for darkness and visual area. Hence, for these two attributes, it appears that a similarity scale generated by the CB model is a magnitude scale. However, the clear rejection of $\mathrm{Cl}$ and Equation 20 shows that neither the ratio estimation scale nor the similarity scale is acceptable if response bias is not taken into account.

Figure 8 gives good support for the CB form of the $\mathrm{J}-\mathrm{J}$ relation for heaviness if it is assumed that the similarity and ratio estimation scales are related by 
a power function. However, Figure 7 indicates conclusively that the ratio estimation and similarity scales do not belong to the same family of magnitude scales $\left(\psi_{\mathrm{s}} \neq \mathrm{K} \psi_{\mathrm{r}}\right)$. This negative result for heaviness is perplexing in view of the positive results for darkness and visual area.

The fits in Figures 5, 6, and 8 are more impressive taking into account that the bias parameters are not estimated via the regression of $S_{a b}^{\prime}$ on $R_{a b}$, but estimated independently in different scaling experiments, and operate as known constants in Equations 21 and 2.5 . The confirmation of the $\mathrm{J}-\mathrm{J}$ relations entailed by the $\mathrm{CB}$ model gives further support to the general judgment model (Equation 14).

The classical psychophysical scaling view considers a response to be a direct reaction to the stimulus. Data from studies reviewed in this paper call for a rejection of this classical view, at least as applied to ratio estimation and the SAM. The classical position is reflected in Equation 2 for ratio estimation and in Equations 1 and 11 for the SAM, and both equations are generally inconsistent with data. The view expressed in this paper is that judgments are mediated by perceived relations of pairs of stimuli (or triples in the case of interval judgments), but that judgments may be biased due to the interactive effect of stimuli, including order effects of various kinds. Equations 2 and 11 are consistent with the relative judgment view only in the absence of response bias. Results with ratio estimation (Fagot, 1978) and unidimensional similarity judgments as reported in this paper are promising for the use of the $C B$ and RB models in the construction of ratio scales of sensation in the presence of response bias. The general model given by Equation 14 shows that the theory can be extended to other judgmental tasks.

\section{REFERENCE NOTF}

1. Franzén, O., Nordmark, J., \& Sjöberg, L. A study of pitch. Göteborg Psychological Reports, University of Göteborg, Sweden, Vol. 2, No. 12, 1972.

\section{REFERENCES}

Adams, E. W., Fagot, R. F., \& Robinson, R. E. On the empirical status of axioms in theories of fundamental measurement. Journal of Mathematical Psychology, 1970, 7, 379-409.
Bartko, J. J. On various intraclass correlation reliability coefficients. Psychological Bulletin, 1976, 83, 762-765.

Bradley, R. A., \& Terhy, M. E. Rank analysis of incomplete block designs. 1. The method of paired comparisons. Biometrika, 1952, 39, 324-345.

Cliff, N. Scaling. Annual Review of Psychology, 1973, 24, 473-506.

Eisl.ER, H. Similarity in the continuum of heaviness with some methodological and theoretical considerations. Scandinavian Journal of Psychology, 1960, 1, 69-81.

Eisle R, H., \& Ekman, G. A mechanism of subjective similarity. Acta Psychologica, 1959, 16, 1-10.

Ekman, G., Engen, T., Kunnapas, T., Lindman, R. A quantitative principle of qualitative similarity. Journal of Experimental Psychology, 1964, 68, 530-536.

Ekman, G., Goude, G., \& Waern, Y. Subjective similarity in two perceptual continua. Journal of Experimental Psychology, 1961, 61, 222-227.

Eкman, G., \& Sü̈веRg, L. Scaling. Annual Review of Psychol$o g y, 1965,16,451-474$.

Fagot, R. F. A theory of relative judgment. Perception \& Psychophysics, 1978, 24, 243-252.

Krantz, D. H. A theory of magnitude estimation and crossmodality matching. Journal of Mathematical Psychology, $1972,9,168-199$.

Kunnapas, T., \& Kunnapas, U. On the relation between similarity and ratio estimates. Psychologische Forschung, 1973, 36, 257-265.

Kunnapas, T., \& Kunnapas, U. On the mechanism of subjective similarity for unidimensional continua. American Journal of Psychology, 1974, 87, 215-222.

Luce, R. D. Individual choice behavior. New York: Wiley, 1959.

Marks, L. E. Sensory processes, the new psychophysics. New York: Academic Press, 1974.

Sлӧвевя, L. Unidimensional similarity revisited. Scandinavian Journal of Psychology, 1966, 7, 115-120.

SJöBERG, L. Three models for the analysis of subjective ratios. Scandinavian Journal of Psychology, 1971, 12, 217-240.

Stevens, S. S., \& Galanter, E. H. Ratio scales and category scales for a dozen perceptual continua. Journal of Experimental Psychology, 1957, 54, 377-411.

\section{NOTES}

1. For the sake of simplicity of notation, $\psi$ is used here to denote the scaling function for both the SAMs and the ratio estimation models. However, this is not intended to imply that the scales are necessarily identical, and, in consideration of judgment-judgment relations below, where scale relations are an issue, the scale functions are distinguished.

2. To insure independence of data points, a method was used that deleted some stimulus triples in the test of $\mathrm{Cl}$, and some stimulus tetrads in the test of C2. See Fagot (1978, footnote 2) for further explanation.

(Received for publication May 14, 1979; revision accepted August 28, 1979.) 